\title{
Practical Methods for Estimating Metabolic Flux (Ki) to Assess Response to Therapy via Static PET Scans
}

\author{
Robert K. Doot \\ Department of Radiology, University of Pennsylvania, Philadelphia, Pennsylvania
}

See the associated article on page 322 .

$\mathbf{I}_{n}$

this issue of The Journal of Nuclear Medicine, Azad et al. compare quantitation of the response of breast cancer bone metastases to therapy via SUV versus metabolic flux (Ki or plasma clearance) using a Ki estimation method that has been designed to ease the clinical burden of data collection from serial ${ }^{18} \mathrm{~F}$-fluoride PET scans (1). This perspective summarizes previous associations of changes in primary breast cancer tumor Ki and SUV with patient outcome from serial ${ }^{18} \mathrm{~F}-\mathrm{FDG}$ PET scans and describes the development of $\mathrm{Ki}$ estimation methods designed to ease the clinical burden of acquiring the data required for traditional $\mathrm{Ki}$ analysis methods.

Overall survival (OS) and disease-free survival (DFS) from locally advanced breast cancer in 75 patients were associated with the posttherapy change in ${ }^{18} \mathrm{~F}-\mathrm{FDG}$ delivery $\left(K_{1}\right.$ : OS, $P=0.005$; DFS, $P=0.001)$ and metabolic flux (Ki: OS, $P=0.006$; DFS, $P=0.01)$ from compartmental analyses of dynamic PET scans, whereas change in SUV $(P=0.02)$ was associated only with OS (OS, $P=0.02$; DFS, $P=0.08$ ) (2). The same study also observed that a prediction model including ${ }^{18} \mathrm{~F}$-FDG delivery and Ki measures had a significantly larger 0.97 area under the receiver-operating-characteristic curve (AUC) than the 0.84 AUC for a model including SUV $(P=0.005)(2)$, indicating that the percentage change in Ki may be more sensitive to measurement of cancer response to therapy than SUV (3). Although more sophisticated kinetic PET measures, such as delivery and $\mathrm{Ki}$, may be more sensitive indicators of response to therapy (3), the need for longer dynamic PET acquisition time and potential requirement for repeated blood sampling are often impractical in a typical PET clinical setting.

Estimation of both PET radiotracer delivery $\left(K_{1}\right)$ and Ki generally requires compartmental kinetic analysis (e.g., Hawkins et al. (4) for analyses of ${ }^{18}$ F-fluoride PET scans) or spectral analysis of a dynamic PET acquisition (5). However, Ki can alternatively be calculated via the graphical Patlak method (6) provided assumptions such as a second irreversible binding compartment are valid for your application. There has been an evolutionary series of

Received Oct. 28, 2018; revision accepted Jan. 15, 2019.

For correspondence or reprints contact: Robert K. Doot, University of Pennsylvania, 160A John Morgan Building, 3620 Hamilton Walk, Philadelphia, PA 19104.

E-mail: robdoot@pennmedicine.upenn.edu

Published online Jan. 25, 2019.

COPYRIGHT (C) 2019 by the Society of Nuclear Medicine and Molecular Imaging. DOI: 10.2967/jnumed.118.220913 modifications to the graphical Patlak method (6) to ease the clinical burden by decreasing the typical 1-h PET acquisition duration or reducing the number of blood samples required for analysis. In 1994, Ishizu et al. proposed the fractional uptake rate (FUR) (7), which replaced the 1-h dynamic acquisition with a single 20-min PET image and calculated the FUR Ki by ignoring the Patlak intercept by assuming the distribution volume is equal to zero. The FUR Ki method had 2 advantages over traditional Patlak Ki: a shorter PET scan duration and the capability to measure $\mathrm{Ki}$ from all areas of a static whole-body PET scan without the limitation of a typical axial field of view of $18 \mathrm{~cm}$ or less. However, the FUR Ki method still required radioactive count data from arterial blood samples drawn from the time of radiotracer injection to the end of the static PET scan. In 1996, Hunter et al. proposed the simplified kinetic method (SKM), which made assumptions similar to the FUR method for approximating the Patlak Ki with the SKM Ki. The SKM method further reduced the clinical burden by replacing the many blood samples required for the FUR method with 1 blood sample (8). This allows estimation of the area under a blood activity curve from a scaled population blood input function, assuming the new input function would have the same shape as in the Hunter et al. studied cohort. The SKM method's requirement to collect blood during the middle of the static scan limits calculation of SKM Ki to a single PET bed position, unlike the FUR Ki method.

Siddique et al. proposed another modified Patlak analysis method for ${ }^{18} \mathrm{~F}$-flouride PET scans of bone regions $(9,10)$. This method replaces the measured blood input function in the Patlak method with a semipopulation blood input function adjusted using 2 venous blood measures, and unlike the FUR and SKM Ki approaches, the Siddique $\mathrm{Ki}$ method retains input from the Patlak intercept using a population mean volume of distribution that varies by bone region $(9,10)$. The Siddique Ki approximation of the Patlak Ki requires 1 venous blood sample taken at least $30 \mathrm{~min}$ after radiotracer injection and before the start of a single static ${ }^{18}$ F-fluoride PET scan and a second venous blood sample after completion of the PET scan $(9,10)$. The Siddique Ki method combines the advantage of the FUR Ki method to estimate Ki from multiple PET scanner bed positions with the advantage of the SKM Ki method of only a limited venous blood sampling requirement. The clinical requirements for the Siddique $\mathrm{Ki}$ method (10) of a single static PET scan acquired between $30 \mathrm{~min}$ to $2 \mathrm{~h}$ after injection and venous blood samples collected before and after the PET scan could make this method practical to perform in any nuclear medicine clinic with the capability to count blood samples. Implementation of this method requires that a semipopulation blood input function and regional volume of distributions have already been determined for the selected radiotracer and study cohort. 
Azad et al. (1) report in this issue on the use the Siddique $\mathrm{Ki}$ method (10) to calculate $\mathrm{Ki}$ for 52 lesions from whole-body ${ }^{18}$ F-fluoride PET scans of 12 breast cancer patients. The static PET scan was acquired $60 \mathrm{~min}$ after radiotracer injection, and 2 venous blood samples were drawn before and after the PET scan at 55 and $85 \mathrm{~min}$ after injection. Percentage changes in Siddique $\mathrm{Ki}, \mathrm{SUV}_{\text {max }}$, and $\mathrm{SUV}_{\text {mean }}$ values from before and after $8 \mathrm{wk}$ of endocrine therapy were compared for 4 patients with clinical progressive disease (PD) within $24 \mathrm{wk}$ and for 8 non-PD patients (up to 5 bone metastases were selected for each subject). Azad et al. reported that the mean $90 \%$ increase in Siddique Ki for the PD patients was significantly greater than the $42 \%$ and $44 \%$ increases in $\mathrm{SUV}_{\max }$ and $\mathrm{SUV}_{\text {mean }}$, respectively $(P<0.001)(1)$. The difference in mean percentage increase in $\mathrm{Ki}$ for $\mathrm{PD}$ versus non-PD patients was significant $(90 \%$ vs. $11 \%, P<0.01)$, whereas the differences in mean percentage increases in $\mathrm{SUV}_{\text {max }}$ and $\mathrm{SUV}_{\text {mean }}$ for PD versus non-PD patients were not $(P \geq 0.07)$. The greater magnitude of reported percentage change in Siddique Ki relative to $\mathrm{SUV}_{\text {mean }}(90 \%$ vs. $44 \%$ ) (1) is consistent with the greater reported magnitude of percentage change in kinetic Ki relative to SUV calculated from dynamic ${ }^{18} \mathrm{~F}$-FDG PET scans of 75 patients with locally advanced breast cancer (2). These reported differences in the magnitudes of Ki versus SUV changes with therapy suggest that percentage changes in Ki from serial ${ }^{18} \mathrm{~F}$-fluoride or ${ }^{18} \mathrm{~F}-\mathrm{FDG}$ may be more sensitive PET measures than SUV for evaluating response to therapy (3) regardless of whether $\mathrm{Ki}$ is calculated via full compartmental kinetic analysis of dynamic PET images (2) or calculated using the Siddique Ki method on static PET images (1). However, a larger prospective clinical trial quantitating patient response to therapy via serial PET scans using both $\mathrm{Ki}$ and SUV is required to determine which PET measure is more predictive of patient outcomes.

\section{DISCLOSURE}

The U.S. National Institute on Drug Abuse of the National Institutes of Health under award number K01DA040023 and the
Susan B. Komen Foundation grant SAC140060 supported this work. No other potential conflict of interest relevant to this article was reported.

\section{ACKNOWLEDGMENTS}

I am thankful for insightful feedback from Erin Schubert, Anthony Young, and $J N M$ reviewers and editors.

\section{REFERENCES}

1. Azad GK, Siddique M, Taylor B, et al. Is response assessment of breast cancer bone metastases better with measurement of ${ }^{18} \mathrm{~F}$-fluoride metabolic flux than with measurement of ${ }^{18}$ F-fluoride PET/CT SUV? J Nucl Med. 2019;60:322327.

2. Dunnwald LK, Doot RK, Specht JM, et al. PET tumor metabolism in locally advanced breast cancer patients undergoing neoadjuvant chemotherapy: value of static versus kinetic measures of fluorodeoxyglucose uptake. Clin Cancer Res. 2011;17:2400-2409.

3. Doot RK, Kurland BF, Kinahan PE, Mankoff DA. Design considerations for using PET as a response measure in single site and multicenter clinical trials. Acad Radiol. 2012;19:184-190.

4. Hawkins RA, Choi Y, Huang SC, et al. Evaluation of the skeletal kinetics of fluorine-18-fluoride ion with PET. J Nucl Med. 1992;33:633-642.

5. Veronese M, Rizzo G, Bertoldo A, Turkheimer FE. Spectral analysis of dynamic PET studies: A review of 20 years of method developments and applications. Comput Math Methods Med. 2016;2016:7187541.

6. Patlak CS, Blasberg RG, Fenstermacher JD. Graphical evaluation of blood-tobrain transfer constants from multiple-time uptake data. J Cereb Blood Flow Metab. 1983;3:1-7.

7. Ishizu K, Nishizawa S, Yonekura Y, et al. Effects of hyperglycemia on FDG uptake in human brain and glioma. J Nucl Med. 1994;35:1104-1109.

8. Hunter GJ, Hamberg LM, Alpert NM, Choi NC, Fischman AJ. Simplified measurement of deoxyglucose utilization rate. J Nucl Med. 1996;37:950955.

9. Siddique M, Blake GM, Frost ML, et al. Estimation of regional bone metabolism from whole-body ${ }^{18}$ F-fluoride PET static images. Eur J Nucl Med Mol Imaging. 2012;39:337-343.

10. Siddique M, Frost ML, Moore AE, Fogelman I, Blake GM. Correcting ${ }^{18} \mathrm{~F}$ fluoride PET static scan measurements of skeletal plasma clearance for tracer efflux from bone. Nucl Med Commun. 2014;35:303-310. 